\title{
Network Coded Modulation for Two-Way Relaying
}

\author{
Wei Chen, Member, IEEE \\ Department of EE, TNList \\ Tsinghua University, China \\ Email: wchen@tsinghua.edu.cn
}

\author{
Lajos Hanzo, Fellow, IEEE \\ School of ECS \\ University of Southampton, UK \\ Email: lh@ecs.soton.ac.uk
}

\author{
Zhigang Cao, Senior Member, IEEE \\ Department of EE, TNList \\ Tsinghua University, China \\ Email: czg-dee@tsinghua.edu.cn
}

\begin{abstract}
Network coding compresses multiple traffic flows with the aid low-complexity algebraic operations, hence holds the potential of significantly improving both the power and bandwidth efficiency of wireless networks. In this contribution, the novel concept of Network Coded Modulation (NCM) is proposed for jointly performing network coding and modulation in bi-directional/duplex relaying. Each receiver is colocated with a transmitter and hence has prior knowledge of the message intended for the distant receiver. As in classic coded modulation, the Euclidian distance between the symbols is maximized, hence the Symbol Error Ratio (SER) is minimized. Specifically, we conceive NCM methods for PSK, PAM and QAM based on modulo addition of the normalized phase or amplitude. Furthermore, we propose low complexity decoding algorithms based on the corresponding conditional minimum distance criteria. Our performance analysis and simulations demonstrate that NCM relying on PSK is capable of achieving a SER at both receivers of the NCM scheme as if the relay transmitted exclusively to a single receiver only. By contrast, when our NCM concept is combined with PAM/QAM, an SNR loss $(<1.25 \mathrm{~dB})$ is imposed at one of the receivers, usually at the one having a lower data rate in a realistic different rate scenario. Finally, we will demonstrate that the proposed NCM is compatible with existing physical layer designs.
\end{abstract}

\section{INTRODUCTION}

Network coding (NC) has emerged as a promising and powerful solution for future communication networks, since it holds the promise of significant throughput gains. The underlying core idea behind network coding is the linear combination and compression of various traffic flows with the aid of low-complexity algebraic operations at intermediate nodes, such as routers or relay stations of a network. These network coded flows will be decoded at their destinations by jointly processing multiple input flows arriving from different paths and/or by exploiting some prior knowledge. This ingenious methodology allows a multicasting communication network to approach its maximum-flow capacity bound [1] and hence to support high-speed multimedia applications, such as IPTV. By exploiting the broadcast nature of wireless downlink (DL) environments, network coding may also attain a substantial power and bandwidth efficiency gain in unicast wireless transmissions.

In the past decade, the theory and application of $\mathrm{NC}$ has attracted extensive investigations. The initial design objective was that of approaching the maximum-flow capacity bound of multicasting wired networks [1]. In an effort to formulate its unified theory and global code construction, linear space based and algebraic frameworks were proposed for single-source multicasting described by a general graph model [2], [3]. In contrast to wired networks, where NC relies on the global network topology, wireless networks are capable of flexibly applying NC for achieving substantial throughput gains for unicast flows, which may be attained without requiring global topology related information. Of particular practical interest is the two-way relay-aided sub-network topology, which relies on bi-directional/duplex relaying. To the best of our knowledge, reference [4] was the first NC-contribution on the practical subject of simultaneous information exchange between two unicast flows, which is also known as bi-directional/duplex relaying. Explicitly, the relay computes the XOR of the two bits received from two distant nodes, which are engaged in bidirectional/duplex communications, and then broadcasts the XORed output bits, which can be decoded at a node by simply XORing the received bit with the bit which was originally transmitted from this node. Assuming the transmission of unbiased random bits, a 50\% potential energy and bandwidth reduction may be achieved at the relay. To broaden the application of wireless network coding, the authors of [5] considered another related sub-network topology, which was referred to as X-relaying.

In both bi-directional and X-relaying, each destination receiver has the prior knowledge of the message intended for the other, which can be expected to simultaneously decode the NC bits or symbols carrying two-way traffic. Hence twoway relaying was extensively studied from both an information theoretic and practical perspective. To understand the performance limits of decode-and-forward aided two-way relaying, its downlink capacity was investigated in [6] and [7]. In particular, [6] proposed a technique for ensuring that the two downlinks can have different channel capacities and hence are capable of asymmetric two-way relaying. As a further advance, the idea of lattice coding was proposed for designing efficient two-way relaying [8]-[10]. Furthermore, NC and modulation were jointly designed for realizing practical asymmetric relaying [10]-[12]. More particularly, a joint NC and superposition coding approach was proposed for a cross-layer operation in [10]. Practical symbol based NC relying on QAM was designed in [11]-[12] for bi-directional transmission.

In this paper, we proposed the novel concept of network coded modulation (NCM) for practical asymmetric two-way relaying. Our main motivation is based on the following three aspects. Firstly, practical duplex relaying needs an efficient and low-complexity transmission strategy for minimizing the 
symbol error ratio (SER) at both receivers. Secondly, the design and analysis of two-way relaying may be substantially simplified, if the NC procedure can be made compatible with existing physical layer techniques, which have been extensively studied. Thirdly, we have to conceive a technique, which is capable of achieving the two-way relaying capacity, in order to attain high coding gains in practical scenarios. Our novel contribution is that NC and modulation are jointly designed in this paper. For PSK, PAM, and QAM, NCM can be realized by the carefully designed modulo addition of the normalized phase or amplitude. We will show that the proposed NCM allows each receiver to decode a constellation that was designed for AWGN channels, where the Euclidian distance of symbols is maximized. In this way, the SER analysis methods conceived for AWGN can be borrowed to show that NCM using PSK can achieve a SER at each of the receivers as if the relay transmitted exclusively to a single receiver only. This confirms the information theoretic conclusions drawn in [6]-[7], namely that each of the twoway downlinks spanning from the relay to the destinations is capable of approaching its AWGN channel capacity. We will also show that NCM using PAM/QAM, rather than PSK, will induce a fixed SNR loss $(<1.25 \mathrm{~dB})$ at one of the receivers, usually at the one receiving at the lower data rate. Finally, we demonstrate that $\mathrm{NCM}$ can be directly combined with most existing physical layer techniques, including TCM as well as Adaptive Modulation and Coding (AMC)[13]. This will substantially reduce the design complexity of two-way relaying.

\section{SySTEM MODEL}

Consider a two-way relaying network, where a common relay transmits to destination nodes $D N_{1}$ and $D N_{2}$, each having a prior knowledge of the message intended for the other, because they also act as source nodes $S N_{1}$ and $S N_{2}$. This scenario is shown in Fig. 1. Throughout this paper, we let $k=3-i$. Let $W_{i}$ denote the message of $S N_{k}$ intended for $D N_{i}, i=1,2$. Thus, when we refer to $D N_{i}$, this reciever has the knowledge of $W_{k}$ intended for $D N_{k}$.

In two-way relaying, the relay node $(R N)$ will transmit a single symbol, which carries the messages to two $D N \mathrm{~s}$ simultaneously. Let $X$ and $Y_{i}$ denote the signal transmitted by the RN and the signals received at $D N_{i}$, respectively. Then the equivalent baseband signal model is presented by

$$
Y_{i}=X+Z_{i},
$$

where $Z_{i}$ denotes the equivalent noise at $D N_{i}$. Furthermore, we have $Z_{i}=\frac{\tilde{Z}_{i}}{h_{i}}$, where $\tilde{Z}_{i}$ represents the Additive White Gaussian Noise (AWGN) at $D N_{i}$, while $h_{i}$ denotes the fading channel coefficient spanning from the $R N$ to $D N_{i}$. Assuming a noise variance of $N_{0}$, we have $\tilde{Z}_{i} \sim \mathcal{C N}\left(0, N_{0}\right)$. In this context, the equivalent noise variance is given by $N_{0, i}=\frac{N_{0}}{\left.h_{i}\right|^{2}}$. Furthermore, let us denote the energy per symbol by $E_{s}:=$ $E\left\{X^{2}\right\}$.

The data rate of the message $W_{i}$ of $S N_{i}$ is denoted by $R_{i}$. Without loss of generality, let us assume $R_{1} \leq R_{2}$ throughout

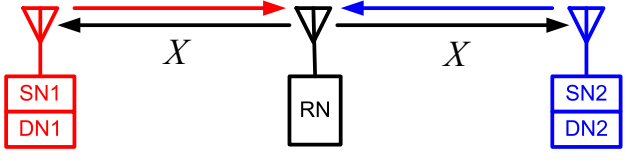

Fig. 1. System Model

this paper. In the symmetric case of $R_{1}=R_{2}$, the RN can perform network coding using bit-by-bit XOR and then map the resultant bits to into symbols. In the above mentioned asymmetric case of $R_{1}<R_{2}$, however, it is suboptimal to use bit-level network coding and modulation separately. Practical asymmetric scenarios occur not only owing to the different transmit requirements of duplex $S N \mathrm{~s}$, but also due to the difference in their link qualities and BER requirements, which may result in different channel coding rates and modulation constellations.

In each symbol duration $T_{s}, R_{1} T_{s}$ bits of $W_{1}$ and $R_{2} T_{s}$ bits of $W_{2}$ are jointly mapped to a symbol $X$ chosen from a set $\mathcal{X}$, which is also referred to as the phaser constellation. In practice, an integer number of bits is mapped to each symbol, i.e. we have $R_{i} T_{s} \in \mathbb{N}$. Conventionally, a constellation that carries $R_{i} T_{s}$ bits/symbol must have $M_{i}=2^{R_{i} T_{s}}$ legitimate symbols. For $R_{1} \leq R_{2}$, we have $\frac{M_{2}}{M_{1}} \in \mathbb{N}$. Having established the system model, we shall propose network coded modulation, which is capable of efficiently transmitting $R_{i} T_{s}$ bits to each receiver $i$ using a single symbol $X$ from a constellation having $M_{2}=2^{R_{2} T_{s}}$ symbols. Notably, the proposed NCM method is also applicable to the symmetric case of $R_{1}=R_{2}$.

\section{Network Coded Modulation}

In this section, we propose and analyze NCM designed for widely used PSK/PAM/QAM constellations, which is realized by the modulo addition of the normalized phase or amplitude of two symbols. We shall propose low-complexity transceiver algorithms and evaluate their SERs. We will also demonstrate for NCM-aided PSK that we can achieve the same SER as if the RN allocated all of its power for transmission to a single DN's receiver using PSK at the same bit rate. By contrast, for network coded PAM/QAM, a fixed SNR loss of less than $1.25 \mathrm{~dB}$ will have to be imposed on the receiver of the lowerrate link of a practical asymmetric scenario.

\section{A. NETWORK CODED PSK}

In network coded PSK, the output symbol, which simultaneously carries two messages, is chosen from a PSK constellation. The RN's transmitter will carry out NCM in two steps per symbol duration. Firstly, $R_{i} T_{s}$ bits of the message $W_{i}$ of $S N_{i}$ will be mapped to a symbol chosen from a normalized $M_{i}$-ary PSK constellation having $M_{i}=2^{R_{i} T_{s}}$ symbols, which are represented by

$$
\mathcal{X}_{i}=\left\{\cos \theta_{i}+j \sin \theta_{i}: \theta_{i} \in \Theta_{i}\right\},
$$

where we have

$$
\Theta_{i}=\left\{0, \frac{2 \pi}{M_{i}}, \ldots, \frac{2\left(M_{i}-1\right) \pi}{M_{i}}\right\} .
$$


Any bit-to-symbol mapping methods, such as Gray coding and set partitioned TCM, can be adopted throughout this paper. Given the phase $\theta_{1}$ and $\theta_{2}$ of the mapped symbols, the RN's transmitter generates a network coded PSK symbol given by

$$
X=\sqrt{E_{s}}(\cos \theta+j \sin \theta),
$$

where the symbol's phase is obtained by

$$
\theta=\theta_{1}+\theta_{2} \bmod 2 \pi \text {. }
$$

The symbols generated by Eqs. (2)-(3) form a $M_{2}$-ary PSK constellation. Note that $\Theta_{i}$ represents a finite group with respect to $\bmod 2 \pi$ addition, and $\Theta_{1}$ is a subset of $\Theta_{2}$. Since the group $\Theta_{2}$ is closed under the considered operation in Eq.(3), the group $\theta$ formed by the resultant phase is equal to $\Theta_{2}$. Moreover, it may be readily shown that $\theta$ is uniformly distributed, if $\theta_{i}$ is uniformly distributed. It can be seen from Eqs. (2)-(3), that the original symbol of link $i$ is rotated by an angle of $\theta_{k}$, which is also known at the receiver of $D N_{i}$ given the prior knowledge of $W_{k}$. In other words, the receiver of $D N_{i}$ will have to detect a rotated PSK constellation. Hence the receivers may apply existing PSK detection methods for our NCM scheme.

Let us now turn our attention to the NCM receiver's symbol detection algorithm. To minimize the SER, the classic maximum a posteriori probability (MAP) detection rule is applied at both DN's receivers. Given the prior knowledge of $\theta_{k}$, the receiver of $D N_{i}$ will detect its desired symbol as

$$
\begin{aligned}
& \hat{\theta}_{i}=\underset{\hat{\theta}_{i} \in \Theta_{i}}{\arg \max } \operatorname{Pr}\left\{\sqrt{E_{s}}\left[\begin{array}{c}
\cos \left(\hat{\theta}_{i}+\theta_{k}\right) \\
+j \sin \left(\hat{\theta}_{i}+\theta_{k}\right)
\end{array}\right] \mid Y_{i}, \theta_{k}\right\} \\
& \stackrel{(a)}{=} \underset{\hat{\theta}_{i} \in \Theta_{i}}{\arg \max } \operatorname{Pr}\left\{Y_{i} \mid \sqrt{E_{s}}\left[\begin{array}{c}
\cos \left(\hat{\theta}_{i}+\theta_{k}\right) \\
+j \sin \left(\hat{\theta}_{i}+\theta_{k}\right)
\end{array}\right]\right\} \\
& \stackrel{(b)}{=} \underset{\hat{\theta}_{i} \in \Theta_{i}}{\arg \min }|| Y_{i}-\sqrt{E_{s}}\left[\begin{array}{c}
\cos \left(\hat{\theta}_{i}+\theta_{k}\right) \\
+j \sin \left(\hat{\theta}_{i}+\theta_{k}\right)
\end{array}\right] \| \\
& \stackrel{(b)}{=} \underset{\hat{\theta}_{i} \in \Theta_{i}}{\arg \min }\left|\angle Y_{i}-\left(\hat{\theta}_{i}+\theta_{k} \bmod 2 \pi\right)\right| \\
&=\underset{\hat{\theta}_{i} \in \Theta_{i}}{\arg \min }\left|\left(\angle Y_{i}-\theta_{k} \bmod 2 \pi\right)-\hat{\theta}_{i}\right|
\end{aligned}
$$

where $\angle Y_{i}$ denotes the phase angle of the received symbol $Y_{i}$. Specifically, equality $(a)$ converts the MAP rule into the Maximum-Likelihood (ML) rule by exploiting the fact that $\theta_{i}$ is uniformly distributed. Equality $(b)$ simplifies the ML rule to the classic minimum Euclidian distance criterion conceived for AWGN channels. For PSK constellations relying purely on phase-rotation, the minimum Euclidian distance criterion is equivalent to the minimum phase difference criterion, as shown in $(c)$. By noting that $\angle Y \in[0,2 \pi)$, we can simply minimize the phase difference between $\angle Y$ and $\left(\hat{\theta}_{i}+\theta_{k} \bmod \right.$ $2 \pi)$. To provide further insights, we may present the analytical expression of $\hat{\theta}_{i}$ as

$$
\hat{\theta}_{i}=\left\lfloor\frac{M_{i}\left(\angle Y_{i}-\theta_{k} \bmod 2 \pi\right)}{2 \pi}+\frac{1}{2}\right\rfloor \frac{2 \pi}{M_{i}},
$$

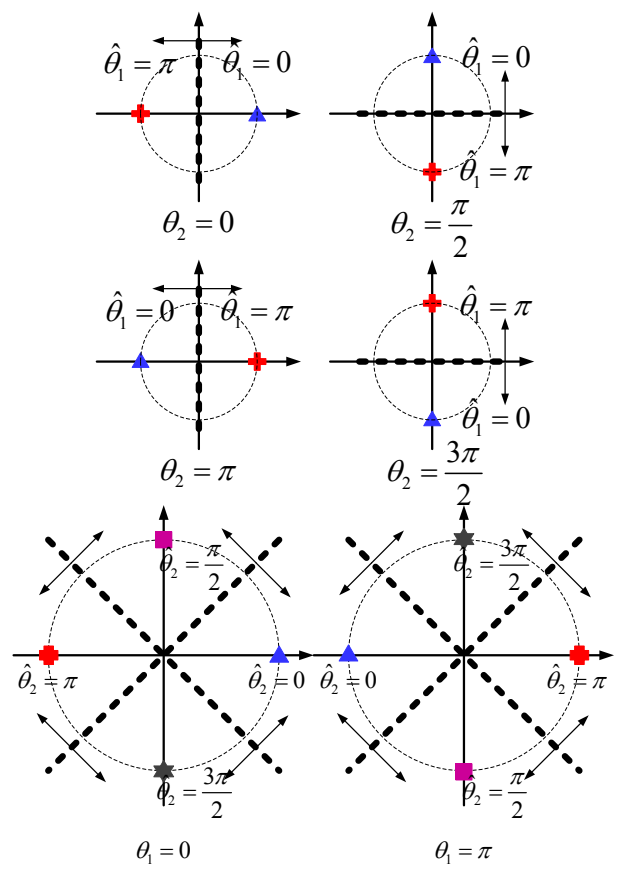

Fig. 2. Decision Regions for Network Coded PSK, $M_{1}=2, M_{2}=4$

or equivalently, if the following condition holds $\frac{2 m \pi}{M_{i}}<$ $\left(\angle Y_{i}+\frac{\pi}{M_{i}}-\theta_{k}\right) \bmod 2 \pi<\frac{2(m+1) \pi}{M_{i}}$, then $\hat{\theta}_{i}=\frac{2 m \pi}{M_{i}}$. From Eqs. (4) and (5), we may infer the decision region required for network coded PSK at the receiver of $D N_{i}$, as shown in Fig. 2 , which can be obtained by rotating the decision region of conventional $M_{i}$-ary PSK by the above-mentioned angle $\theta_{k}$. This implies that the detection complexity of network coded PSK is the same as that of common PSK.

Having conceived the transceiver scheme of network coded PSK, let us now study its SER performance. Recall from the above discussions that both the constellation and decision regions of network coded PSK are rotated versions of conventional $M_{i}$-ary PSK with a symbol energy of $E_{s}$. Intuitively, given the same symbol distances and decision rules, the network coded PSK and conventional $M_{i}$-ary PSK should have the same SER, as shown in the following theorem.

Theorem 1: The SER at the receiver of $D N_{i}$ can be obtained by

$$
P_{i}= \begin{cases}Q\left(\sqrt{\frac{2 E_{s}}{N_{0, i}}}\right) & M_{i}=2 \\ 2 Q\left(\sqrt{\frac{E_{s}}{N_{0, i}}}\right)-Q^{2}\left(\sqrt{\frac{E_{s}}{N_{0, i}}}\right) & M_{i}=4 \\ 2 Q\left(\sqrt{\frac{2 E_{s}}{N_{0, i}}} \sin \frac{\pi}{M_{i}}\right) & M_{i}>4 .\end{cases}
$$

Proof: Since $\theta_{i}$ is uniformly distributed in $\Theta_{i}$, the average SER is equal to the symbol error probability conditioned on $\theta_{i}=0$ and $\theta_{k}=0$ for reasons of symmetry. As a result, we have

$$
\begin{aligned}
P_{i} & =\operatorname{Pr}\left\{\hat{\theta}_{i} \neq 0 \mid \theta_{i}=0, \theta_{k}=0\right\} \\
& =2 \operatorname{Pr}\left\{\left|\angle\left[\sqrt{E_{s}+\operatorname{Re}\left\{Z_{i}\right\}}+j \operatorname{Im}\left\{Z_{i}\right\}\right]\right| \geq \frac{\pi}{M_{i}}\right\} .
\end{aligned}
$$


From [14], we arrive at the SER expression $P_{i}$ of (6) at the receiver of $D N_{i}$.

Based on Theorem 1, network coded PSK achieves the same $\mathrm{SER}$ at the receiver of the $\mathrm{DN}$, as if the $R N$ exclusively allocated all of its power for transmission to a single receiver only. This implies the optimality of network coded PSK. Furthermore, this conclusion is in line with the information theoretic conclusions drawn in [6] and [7], where both links were shown to achieve their AWGN channel capacities in the context of two-way relaying.

\section{B. NETWORK CODED PAM/QAM}

Let us now extend our attention to higher-throughput constellations, such as PAM and QAM. Note that the In-phase $(I)$ and Quadrature $(Q)$ components of rectangular $M$-QAM using $M=L^{2}$ can be regarded as a pair of $L$-PAM signals, because we mainly focus our attention on PAM. Network coded PAM is implemented in two steps per symbol duration. First, $R_{i} T_{s}$ bits of $W_{i}$ will be mapped to a symbol from the set of $M_{i}$-ary PAM constellation points, which is formulated as:

$$
\mathcal{X}_{i}=\left\{2 M_{i} a_{i}-\left(M_{i}-1\right): a_{i} \in \mathcal{A}_{i}\right\},
$$

where

$$
\mathcal{A}_{i}=\left\{0, \frac{1}{M_{i}}, \ldots, \frac{M_{i}-1}{M_{i}}\right\} .
$$

Given the normalized amplitudes $a_{1}$ and $a_{2}$, the $R N$ 's transmitter will generate the network coded PAM symbol as

$$
X=d\left[2 M_{2} a-\left(M_{2}-1\right)\right],
$$

where the normalized amplitude is determined by

$$
a=a_{1}+a_{2} \bmod 1,
$$

and $d=\sqrt{\frac{3 E_{s}}{M_{2}^{2}-1}}$ denotes half of the symbol distance in PAM, given an energy $E_{s}$ per symbol.

Similar to our arguments in Section III-A, the normalized amplitude of the network coded PAM symbol is uniformly distributed in $\mathcal{A}_{2}$. Furthermore, when network coded PAM is adopted, the original symbol intended for $D N_{i}$ will be cyclically shifted by an amplitude of $2 M_{2} a_{k} d$. Again, the receiver of $D N_{i}$ which knows $W_{k}$ a priori and will detect a cyclically shifted PAM constellation. Therefore, both the detection method and SER analysis of conventional PAM can be borrowed for network coded PAM.

The MAP detection method of network coded PAM at $D N_{i}$ is presented as follows:

$$
\begin{aligned}
\hat{a}_{i} & =\underset{\hat{a}_{i} \in \mathcal{A}_{i}}{\arg \max } \operatorname{Pr}\left\{d\left[\begin{array}{c}
2 M_{2}\left(\hat{a}_{i}+a_{k} \bmod 1\right) \\
-\left(M_{2}-1\right)
\end{array}\right] \mid Y_{i}, a_{k}\right\} \\
& \stackrel{(a)}{=} \underset{\hat{a}_{i} \in \mathcal{A}_{i}}{\arg \max } \operatorname{Pr}\left\{Y_{i} \mid d\left[\begin{array}{c}
2 M_{2}\left(\hat{a}_{i}+a_{k} \bmod 1\right) \\
-\left(M_{2}-1\right)
\end{array}\right]\right\} \\
& \stackrel{(b)}{=} \underset{\hat{a}_{i} \in \mathcal{A}_{i}}{\arg \min }\left|Y_{i}-d\left[\begin{array}{c}
2 M_{2}\left(\hat{a}_{i}+a_{k} \bmod 1\right) \\
-\left(M_{2}-1\right)
\end{array}\right]\right| \\
& \stackrel{(c)}{=} \underset{\hat{a}_{i} \in \mathcal{A}_{i}}{\arg \min }\left|\frac{Y_{i}+M_{2}-1}{2 M_{2} d}-\left(\hat{a}_{i}+a_{k} \bmod 1\right)\right|
\end{aligned}
$$

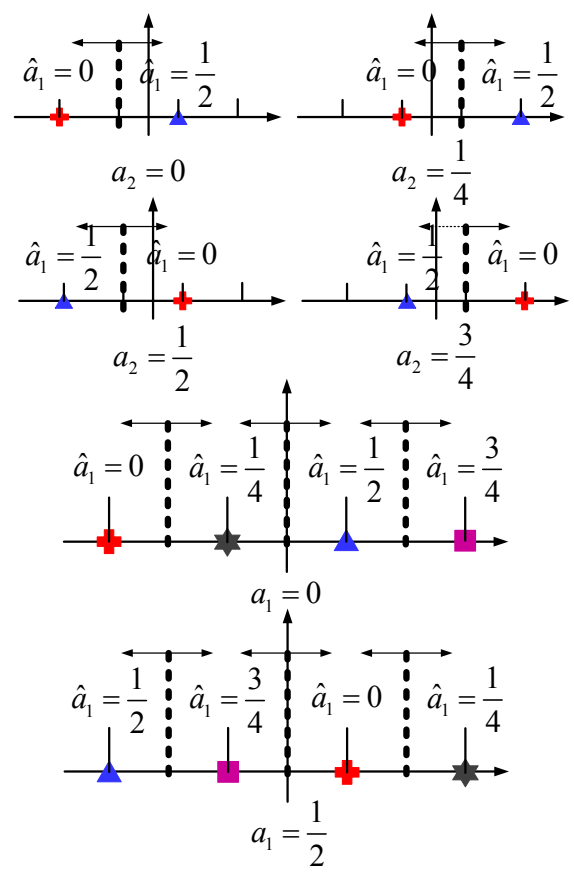

Fig. 3. Decision Regions for Network Coded PAM, $M_{1}=2, M_{2}=4$

Specifically, equalities (a) and (b) yield the ML rule and the minimum Euclidian distance criterion, respectively. For convenience, we normalized the minimum distance criterion, as shown in equality $(c)$. Based on Eq. (9), the analytical expression of the estimated value $\hat{a}_{i}$ of $a_{i}$ in Eq.(8) can be formulated as

$\hat{a}_{i}= \begin{cases}1-a_{k} \bmod 1 & \tilde{Y}_{i}<\underline{\lambda}\left(a_{k}\right)\left(a_{k}\right) \\ \left\lfloor M_{i}\left(\tilde{Y}_{i}-a_{k} \bmod 1\right)+\frac{1}{2}\right\rfloor \frac{1}{M_{i}} & \text { otherwise } \\ a_{k}-\frac{1}{M_{i}} \bmod 1 & \tilde{Y}_{i}>\bar{\lambda}\left(a_{k}\right),\end{cases}$

where $\tilde{Y}_{i}=\frac{Y_{i}+M_{2}-1}{2 M_{2} d}$ is the normalized decision variable. The two extreme boundaries are given by

$$
\begin{aligned}
& \underline{\lambda}\left(a_{k}\right)=\left(a_{k} \bmod \frac{1}{M_{i}}\right)+\frac{1}{2 M_{i}} \\
& \bar{\lambda}\left(a_{k}\right)=\left(a_{k} \bmod \frac{1}{M_{i}}\right)+1-\frac{3}{2 M_{i}}
\end{aligned}
$$

respectively. Fig. 3 shows the decision region of network coded PAM, which is a cyclically shifted version of conventional PAM, as expected. By exploiting the connection between network coded PAM and classic PAM, we formulate its SER in the following theorem.

Theorem 2: The SERs at the receivers of $D N_{1}$ and $D N_{2}$ can be expressed as

$$
\begin{aligned}
& P_{1}=\frac{2\left(M_{1}-1\right)}{M_{1}} Q\left(\frac{M_{2}}{M_{1}} \sqrt{\frac{6}{M_{2}^{2}-1} \frac{E_{s}}{N_{0,1}}}\right) \\
& P_{2}=\frac{2\left(M_{2}-1\right)}{M_{2}} Q\left(\sqrt{\frac{6}{M_{2}^{2}-1} \frac{E_{s}}{N_{0,2}}}\right)
\end{aligned}
$$


Proof: Given the knowledge of $a_{k}$, the receiver of $D N_{i}$ will detect the symbol from a cyclically shifted $M_{i}$-ary PAM constellation. Intuitively, the SER of a cyclically shifted $M_{i}$ ary PAM constellation is identical to that of the original $M_{i^{-}}$ ary PAM for the same minimum symbol distance. From [14], $M_{i}$-ary PAM using a minimum symbol distance of $2 d_{i}$ has the SER given by

$$
P_{i}=\frac{2\left(M_{i}-1\right)}{M_{i}} Q\left(\sqrt{\frac{2 d_{i}^{2}}{N_{0, i}}}\right) .
$$

By recalling Eqs. (7)-(8) and that $a_{i} \in \mathcal{A}_{i}$, we have $d_{1}=\frac{M_{2}}{M_{1}} d$ and $d_{2}=d$, which is inserted into (13) to arrive at Eqs. (11)(12).

Let us now compare the SERs of network coded PAM and classic PAM to provide further insights. The $R N-D N_{2}$ link can achieve the same SER, as if the relay transmitted exclusively to $D N_{2}$. By contrast, the $R N-D N_{1}$ Link suffers from an SNR loss compared to the classic PAM. To understand this further, let $E_{s}^{\prime}$ denote the symbol energy of classic PAM. Then, the SER of classic $M_{1}$-ary PAM is equal to that of network coded PAM, as shown in Eq. (11). The comparison of Eq. (11) and the SER formula of $M_{1}$-ary PAM in [14] suggests that we have

$$
\frac{E_{s}}{E_{s}^{\prime}}=\frac{1-M_{2}^{-2}}{1-M_{1}^{-2}}<\frac{1}{1-M_{1}^{-2}},
$$

which is fixed and independent of the SNR. By noting that $M_{i} \geq 2, \frac{E_{s}}{E_{s}^{\prime}}$ is upper bounded by $\frac{4}{3}$. In other words, the SNR loss due to NCM will be less than $1.25 \mathrm{~dB}$. Furthermore, Eq. (14) also implies that the SNR loss due to NCM becomes negligible, when $M_{i}$ is large. For instance, for $M_{1}=8$, the SNR loss of the $R N-D N_{1}$ link is within $0.069 \mathrm{~dB}$. The reason for the SNR loss at the receiver of $D N_{1}$ can be stated as follows. Given $a_{2}$, the legitimate symbols at the receiver of $D N_{1}$ have a nonzero mean of $d\left[2 M_{2}\left(a_{2} \bmod \frac{1}{M_{1}}\right)+1-\frac{M_{2}}{M_{1}}\right]$. In contrast to the classic zero-mean $M_{i}$-ary PAM, the DC bias of such a cyclically shifted $M_{i}$-ary PAM constellation will cost some extra energy, which therefore results in the above mentioned SNR loss.

Recall that rectangular $L^{2} \mathrm{QAM}$ can be regarded as a pair of $L$-ary PAM $I$ and $Q$ components, each having $\frac{E_{s}}{2}$ energy per symbol. In this context, network coded QAM can be realized by adopting network coded PAM in the $I$ and $Q$ component respectively. Moreover, note that a symbol detection error occurs in QAM if and only if an error occurs in either one of its components. As a result, we may apply the SER results of network coded PAM for expressing the SER of network coded QAM as follows:

$$
\begin{aligned}
& P_{1}=\frac{4 M_{1}-4 \sqrt{M_{1}}}{M_{1}} Q\left(\sqrt{\frac{M_{2}}{M_{1}} \frac{3}{\left(M_{2}-1\right)} \frac{E_{s}}{N_{0,1}}}\right) \\
& P_{2}=\frac{4 M_{2}-4 \sqrt{M_{2}}}{M_{2}} Q\left(\sqrt{\frac{3}{\left(M_{2}-1\right)} \frac{E_{s}}{N_{0,2}}}\right) .
\end{aligned}
$$
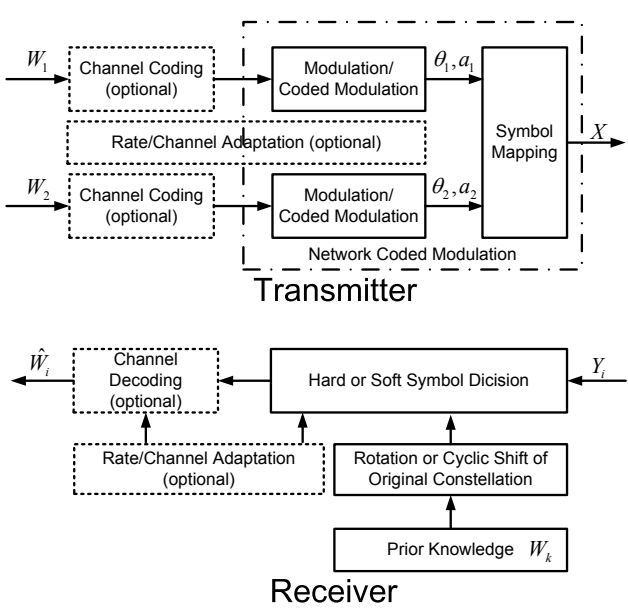

Fig. 4. NCM compatible with existing PHY designs

Similar to our arguments for network coded PAM, network coded QAM will induce a fixed SNR loss in the receiver of $D N_{1}$, which is upper-bounded by $1.25 \mathrm{~dB}$.

Finally, in this section we offer some remarks on the compatibility and optimality of NCM. Fig. 4 presents a solution for ensuring the compatibility of NCM with arbitrary existing physical layer designs. In the $R N$ 's transmitter, NCM will be adopted to process two normalized symbols, both of which are obtained by conventional coding/modulation or coded modulation employed for each flow, and then mapped to a single symbol having an average energy of $E_{s}$. By exploiting the prior knowledge $W_{k}$ at $\mathrm{SN} i$, the receiver of $D N_{i}$ becomes capable of decoding the transmitted symbols of $\mathrm{SN} i$, which are from a rotated PSK or cyclically shifted PAM/QAM constellation. Based on the minimum distance criterion, the decision region will be obtained by rotating or cyclically shifting the original decision region. Although the focus of this paper is on hard decisions, NCM is also compatible with soft decision methods, where the required soft-values can be calculated based on the Euclidian distances between the noisy received symbol and the legitimate symbol. As a result, both the performance analysis as well as the channel-quality-aided adaptation methods of existing physical layer designs can be directly applied.

Recall that network coded PSK is capable of achieving the same symbol distances at both $D N \mathrm{~s}$, as if the relay transmitted exclusively to a single receiver. The same conclusion is true for NCM using PAM/QAM at the high rate receiver. For the lower rate PAM/QAM message receiver, the SNR loss of network coded PAM is less than $0.28 \mathrm{~dB}$ for $M_{1} \geq 4$. This implies the optimality of network coded PSK and near-optimality of network coded PAM/QAM. The optimality formulated in terms of symbol distance and SER was discussed in parallel to the optimality in terms of capacity in [6], [7], suggesting that both the $R N-D N_{1}$ and $R N-D N_{2}$ links are capable of approaching their own single link capacity. However, it is worth noting that we cannot directly apply the bit-tosymbol mapping method proposed in [6], which relies on the 


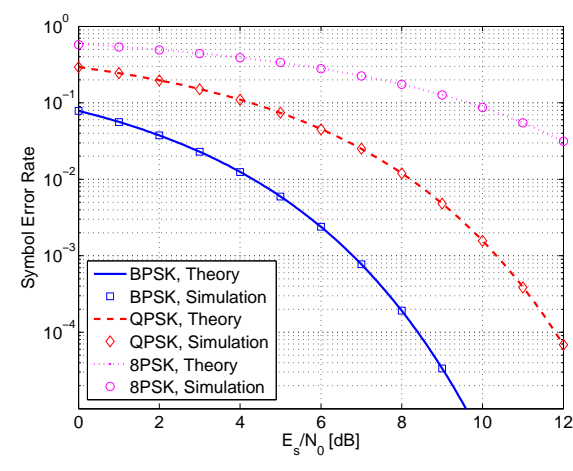

Fig. 5. SER of Network Coded PSK

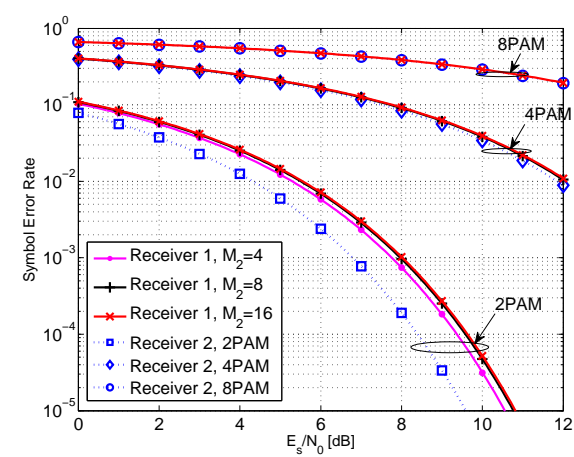

Fig. 6. SER of Network Coded PAM modulo $M_{2}$ addition of the symbol indices, while avoiding any decoding ambiguity, because Xie's solution cannot maximize the Euclidian distances of legitimate symbols, while using a finite code length. By contrast, upon using the modulo addition of the normalized phase or amplitude, the proposed NCM becomes capable of maximizing the Euclidian distances of the legitimate symbols at both $D N_{1}$ and $D N_{2}$.

\section{Simulation Results}

In this section, we shall present our theoretical and simulation-based SER results to demonstrate the potential of the proposed network coded modulation and to validate our theoretical analysis. Observe in Fig. 5 that the theoretical SER of network coded PSK obtained in Eq.(6) and the simulationbased results match well. Again, network coded PSK achieves the same SER at the receiver of the $D N$, as if the $R N$ transmitted exclusively to the single $D N$ at a same SNR. This verifies the optimality of network coded modulation for PSK constellations. By contrast, the SER of network coded PAM is presented in Fig. 6. Similarly, the simulation results can validate the theoretical SER results of Eqs. (11)-(12). In contrast to network coded PSK, NCM relaying on PAM constellations may impose some SNR loss compared to classic one-way transmission using the same constellation. It can be seen that the SNR loss of link 1 is an increasing function of $M_{2}$, but a deceasing function of $M_{1}$. Quantitatively, for $M_{1}=4$, the SNR loss of $D N_{1}$ is less than $0.28 \mathrm{~dB}$, which is negligible in practice. Therefore, one may also conclude that network coded modulation is near-optimal for high order MPAM constellations.

\section{CONCLUSION}

In this paper, NCM was proposed for symmetric and asymmetric two way relaying using PSK and PAM/QAM signalling, which was carried out using the modulo addition of the normalized phase or amplitude of the original symbols intended for $D N_{1}$ and $D N_{2}$ after bit-to-symbol mapping from the $R N$ $D N$ messages. It was shown that both the constellations and the decision regions are rotated or cyclically shifted versions of the original PSK or PAM/QAM scheme, respectively. Based on this observation, we evaluated the SER of network coded PSK, which is exactly the same as that of classic single-link PSK. The SER of network coded PAM/QAM is also identical to that of classic single-link PAM/QAM in the $R N-D N_{2}$ link, but it has a modest SNR loss in the $R N-D N_{1}$ link having a lower message rate. Noting the similarity of NCM and conventional single link transmission schemes, we made it plausible that NCM is compatible with existing physical layer techniques, which makes two-way relaying an effective design option.

\section{REFERENCES}

[1] R. Ahlswede, N. Cai, S. -Y. R. Li and R. W. Yeung, "Network information flow," IEEE Trans. Inform. Theory, vol. 46, pp. 1204 - 1214, Jul 2000.

[2] S. -Y. R. Li, R. W. Yeung and N. Cai, "Linear network coding," IEEE Trans. Inform. Theory, vol. 49, pp. 371 - 381, Feb 2003.

[3] R. Koetter and M. Medard "An algebraic approach to network coding," IEEE/ACM Trans. Networking, vol. 11, pp. 782 - 795, Oct 2003.

[4] Y. Wu, P. A. Chou and S.-Y. Kung, 'Information exchange in wireless networks with network coding and physical-layer broadcast," Microsoft Research Technical Report MSR-TR -2004-78, Aug 2004.

[5] S. Katti, H. Rahul, W. Hu, D. Katabi, M. Medard, and J. Crowcroft, "XORs in the air: practical wireless network coding," IEEE/ACM Trans. on Networking, vol. 16, pp. 497-510, June 2008.

[6] L. -L. Xie, "Network coding and random binning for multi-user channels," Proceedings of 10th Canadian Workshop on Information Theory, Edmontin, Alberta, pp.85-88, June, 2007.

[7] Y. Wu, "Broadcasting when receivers know some messages a priori" Proceedings of IEEE International Symposium on Information Theory, Nice, France, pp. 1141 - 1145, June, 2007.

[8] K. Narayanan, M. P. Wilson, and A. Sprintson. "Joint physical layer coding and network coding for bi-directional relaying," Proceedings of Allerton Conference on Communication, Control and Computing, Monticello, IL, USA, 2007.

[9] I.-J. Baik and S.-Y. Chung, "Network coding for two-way relay channels using lattices," Proceedings of IEEE International Conference on Communications, Beijing, China, pp. 3898 - 3902, May, 2008.

[10] J. Sykora and A. Burr, "Network coded modulation with partial sideinformation and hierarchical decode and forward relay sharing in multisource wireless network," Proceedings of European Wireless Conference, Tuscany, Italy, pp. 639 - 645, April, 2010.

[11] J. M. Park, S.-L. Kim, and J. Choi, "Hierarchically modulated network coding for asymmetric two-way relay systems," IEEE Transactions on Vehicular Technology, vol. 59, pp. 2179-2184, June, 2010.

[12] R. Y. Kim and Y. Y. Kim, "Symbol-level random network coded cooperation with hierarchical modulation in relay communication," IEEE Transactions on Consumer Electronics, vol. 55, pp. 1280-1285, August, 2009.

[13] L. Hanzo, et al, Quadrature Amplitude Modulation: From Basics to Adaptive Trellis-Coded, Turbo-Equalised and Space-Time Coded OFDM, CDMA and MC-CDMA Systems, 2nd ed. Wiley-IEEE Press, 2004.

[14] J. G. Proakis, Digital Communications, 5th ed. New York: McGraw-Hill, 2007. 